\title{
Detection and analysis of apoptosis in peripheral blood cells from breast cancer patients
}

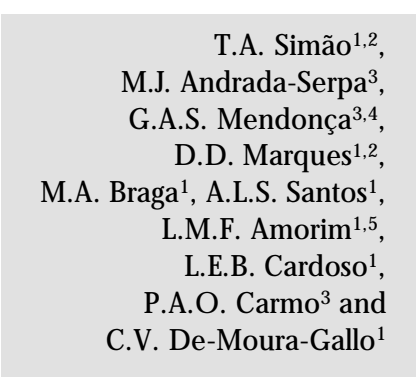

\author{
${ }^{1}$ Departamento de Bioquímica, Instituto de Biologia Roberto Alcantara Gomes, \\ Universidade do Estado do Rio de Janeiro, Rio de Janeiro, RJ, Brasil \\ 2Departamento de Bioquímica, Universidade do Rio de Janeiro, Rio de Janeiro, \\ RJ, Brasil \\ ${ }^{3}$ Setor de Pesquisa Básica, Instituto Nacional de Câncer, Rio de Janeiro, RJ, Brasil \\ ${ }^{4}$ Instituto de Medicina Social, Universidade do Estado do Rio de Janeiro, \\ Rio de Janeiro, RJ, Brasil \\ ${ }^{5}$ Departamento de Biologia Celular e Molecular, Universidade Federal Fluminense, \\ Rio de Janeiro, RJ, Brasil
}

\section{Correspondence \\ C.V. De-Moura-Gallo \\ Departamento de Bioquímica IBRAG, UERJ \\ Av. 28 de Setembro, 87, fundos 40 andar \\ 20551-013 Rio de Janeiro, RJ \\ Brasil \\ Fax: +55-21-587-6136 \\ E-mail: cgallo@uerj.br}

Presented at the XIII Annual M eeting of the Federação de Sociedades de Biologia Experimental, Caxambu, MG, Brasil, August 26-29, 1998.

Research supported by UERJ, CNPq and INCa.

Received April 14, 1998 Accepted January 18, 1999

\section{Abstract}

Apoptosis is a well-known specific process of cell death that normally occurs in physiological situations such as tissue or organ development and involution. During tumor growth there is a balance between proliferation and cell death which involves apoptotic mechanisms. In the present study genomic DNAs from 120 breast tumor biopsies were analyzed by agarose gel electrophoresis and none of them presented the fragmentation pattern characteristic of the apoptosis process. However, $33 \%$ of the 105 breast cancer patients clearly showed the apoptotic pattern when DNA from blood cells was analyzed. None of the DNAs from healthy volunteer blood cells showed any trace of apoptosis. Since the breast cancer patients were not receiving chemoor hormone therapy, the possible relationship between blood cortisol levels and the apoptotic pattern found in patient blood cells was investigated. Using a chemoluminescence immunodetection assay, similar cortisol levels were observed in breast cancer patient sera presenting or not apoptotic blood cells and in healthy volunteer sera. Analysis of the clinical data obtained from 60 of these patients showed that patients bearing tumors of smaller size (under $20 \mathrm{~mm}$ ) were more susceptible to the apoptotic effect in blood cells. According to the Elston grade, it was observed that 7 of 12 patients with grade III tumors (58\%) presented apoptotic peripheral blood cells, in contrast to 10 of 48 patients with grade I and grade II tumors. These observations may reflect the immunosuppression characteristic of some breast cancer patients, which may contribute to tumor growth. Therefore, further studies are necessary to elucidate the factor(s) involved in such massive blood cell death.
Key words

- Apoptosis

- Breast cancer

- Peripheral blood cells

- Cortisol

- Fas-system 
Figure 1 - Agarose gel electrophoresis of peripheral blood cell DNA obtained from breast cancer patients (panel A) and from healthy volunteers (panel $B$ ). The genomic DNAs were extracted from peripheral blood samples collected in the morning in the presence of EDTA. The blood sample was treated with an erythrocyte lysis solution (155 $\mathrm{mM} \mathrm{NH}_{4} \mathrm{Cl}, 10 \mathrm{mM} \mathrm{KHCO}_{3}$ and $1 \mathrm{mM}$ EDTA, pH 7.4) and then with extraction buffer $(10 \mathrm{mM}$ Tris- $\mathrm{HCl}, 100 \mathrm{mM} \mathrm{NaCl}, 25 \mathrm{mM}$ EDTA, $0.5 \%$ SDS and $0.1 \mathrm{mg} / \mathrm{ml}$ proteinase $\mathrm{K}, \mathrm{pH} 8.2$ ) at $57^{\circ} \mathrm{C}$ overnight. The DNA was precipitated with $1.5 \mathrm{M} \mathrm{NaCl}$ solution and ethanol. After washing with $70 \%$ ethanol, the preparation was resuspended in water and the DNA concentration was obtained by measuring absorbance at $260 \mathrm{~nm}$. Three micrograms of the DNA was applied to a $1 \%$ agarose gel, using TBE $(0.09 \mathrm{M}$ Tris-borate, 2 mM EDTA, pH 8.3) as electrophoresis buffer. Electrophoresis was performed at approximately $5 \mathrm{~V} / \mathrm{cm}$. The gels were stained with $1 \mathrm{mg} / \mathrm{ml}$ ethidium bromide and viewed under UV light. Note the characteristic apoptotic pattern present in the peripheral blood cells from breast cancer patients in the lanes presented in panel $A$ and indicated by the arrows.
Apoptosis is a phenomenon that mediates physiological processes such as embryogenesis, metamorphosis, endocrine-dependent tissue atrophy and normal tissue turnover (1). In the immune response, "life and death" lymphocytes are controlled by cytolytic mechanisms resulting from a tightly regulated process of apoptotic cell death (2).
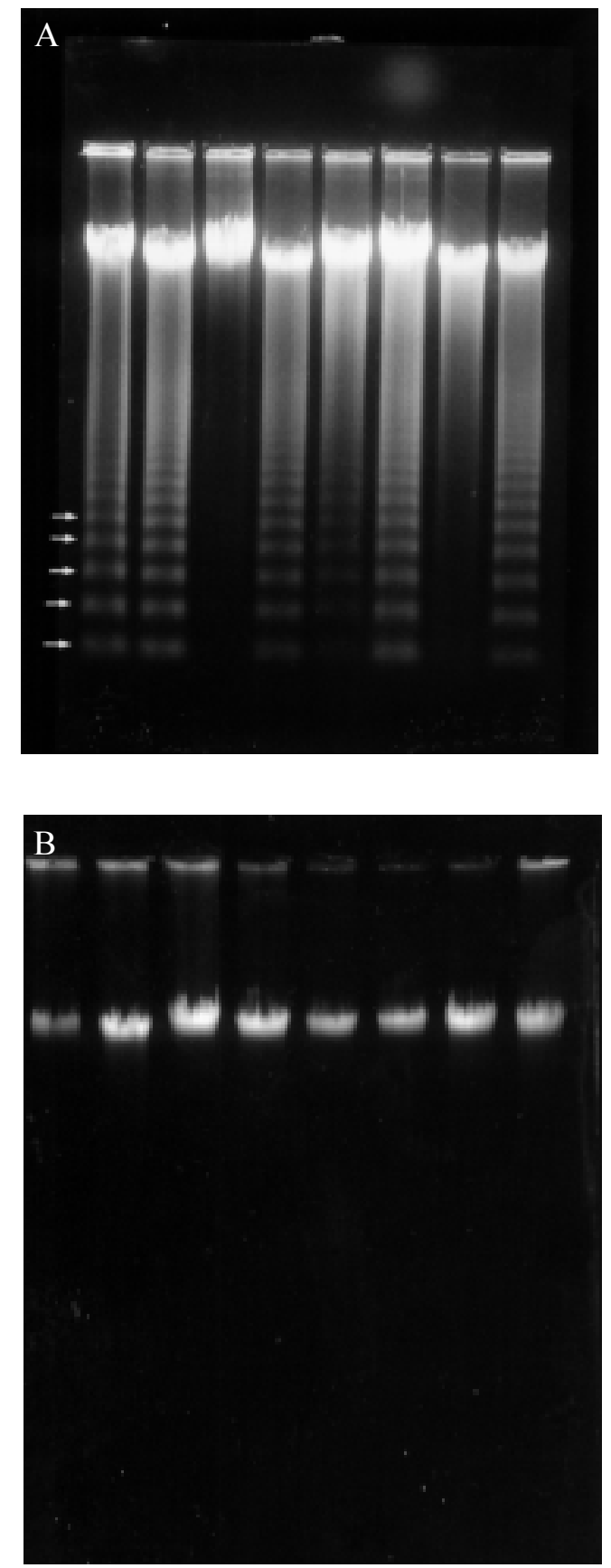

The apoptotic process can be triggered by an intracellular mechanism that seems to be dependent on the $\mathrm{p} 53$ protein, or by external factors such as hormones, cytokines and anti-tumor drugs. Glucocorticoids represent a class of hormones that can trigger cell death. It seems that their role is downregulating the immune response in order to reduce the deleterious consequences of this process, such as inflammatory reactions (3). Apoptosis inducers include the cytokines FasL and TNF, known as "death factors" (1). TNF was first described as cachectin, a factor produced by cancer patients which is responsible for the "cachectic" state as a result of systemic tissue damage (4). The mechanism of TNF action differs from that related to the Fas system. Both factors act through their specific receptors. The Fas system depends on the ability of FasL, a membrane protein mainly expressed in activated $T$ lymphocytes, to bind its receptor Fas (APO-1 or CD95), also a membrane protein. The result of this process is a cascade of events that culminate in the target cell death (1). On the other hand, the action of a variety of antitumor drugs such as cisplatin, a widely used anticancer drug, is based on the induction of apoptosis (5).

Apoptosis frequently occurs in human tumors and seems to be a significant component of the continuous cell loss that usually takes place during tumor formation (6). In an attempt to determine if tumor cells from breast cancer are under control of apoptosis, genomic DNA was extracted from 120 breast tumor biopsies obtained from patients diagnosed at the National Cancer Institute of Brazil (INCa). This study was approved by the Ethics Committee of INCa and patients gave their informed consent. The patients included in the study received no chemotherapy or hormone therapy. DNA analysis by agarose gel electrophoresis proved that none of the tumors analyzed showed the characteristic pattern of DNA fragmentation (data not shown). The investigation of a 
systemic effect was possible by the same analysis using peripheral blood cells from the patients mentioned above. With this purpose the genomic DNA was extracted from both breast cancer patients and healthy volunteer blood samples. Figure 1 represents an agarose gel electrophoresis of representative patients (panel A) and healthy volunteers (panel B), where the DNA fragmentation profile is quite clear. Thirty-five of 105 patients $(33 \%)$ presented such profile which is a hallmark of the apoptosis process, while none of the blood samples from 57 healthy individuals showed this pattern. The observation of the apoptotic profile in patient blood cells (Figure 1, panel A) suggests that these cells might be dying massively.

The clinical data for 60 patients from the group described above were analyzed. Ninety-two percent of these tumors were diagnosed as invasive ductal carcinoma and the other $8 \%$ were distributed between medullary, tubular and mixed carcinoma. This subgroup presented almost the same proportion of patients with apoptotic peripheral blood cells. Lymph node status and age did not show any relation with the presence of apoptosis in the peripheral blood cells as demonstrated by the Fisher exact test. A significant difference in the number of patients presenting apoptosis or not was found in relation to tumor size $(\mathrm{P}=0.0399)$. $\mathrm{Pa}-$ tients bearing tumors of smaller size (under $20 \mathrm{~mm}$ ) were more susceptible to the apoptotic effect. Interestingly, the histological analysis of tumor grade (Elston grade) showed a good relation between grade III tumors and the number of patients presenting apoptotic peripheral blood cells ( $\mathrm{P}=$ $0.0267)$. Seven of 12 patients $(58 \%)$ with grade III tumors were positive for the presence of apoptosis in blood cells. The results are shown in Table 1. The Elston grade corresponds to the tumor aggressiveness and is correlated with patient survival (7). Patients with grade I tumors have better survival than those with grade II and III.
In order to better understand the phenomenon, cortisol concentration in blood samples was determined, since this is a hormone produced by the adrenals and released into the blood stream during a variety of stimuli, including stress. Cortisol is a glucocorticoid able to trigger apoptotic cell death by binding to its specific receptors on target cells. This fact is very important in the treatment of patients with hypereosinophilia who show a rapid and relevant reduction in eosinophil number after glucocorticoid administration (3).

The peripheral blood of 15 patients of the total 105 cited above, presenting or not the apoptotic pattern, and the blood of 11 healthy volunteers were collected at approximately the same time in the morning. Cortisol was detected in the sera using the Immulite chemoluminescence kit. This kit is widely used in hospitals as an immunodetection assay, and is highly sensitive for measuring cortisol concentration. The cortisol levels from patient sera presenting or not apoptotic blood cells were not higher than those observed in normal individuals ( $\mathrm{P}>0.05)$. Normal blood cortisol levels are 2.5 to $12.5 \mu \mathrm{g} /$ $\mathrm{dl}$ and none of the values obtained were higher than this concentration (data not shown). These results indicate that the massive apoptosis previously observed in pe-

Table 1 - Number of patients presenting or not apoptotic peripheral blood cells in relation to tumor size and grade.

alnformation not available for 2 cases. bDetermined by the Fisher exact test.

\begin{tabular}{lrrr}
\hline \multirow{2}{*}{ Tumor features } & \multicolumn{2}{c}{ Apoptosis } & $\mathrm{Pb}$ \\
\cline { 2 - 3 } & Negative & Positive & \\
\hline Sizea $(\mathrm{mm})$ & & & \\
$\leq 20$ & 6 & 7 & 0.0399 \\
$>20$ & 35 & 10 & \\
Elston grade & & & \\
$\quad \triangleleft$ II & 38 & 10 & \\
III & 5 & 7 & 0.0267 \\
& & &
\end{tabular}


ripheral blood cells of breast cancer patients is not related to increased cortisol levels.

Cancer patients may produce TNF $\alpha$ in soluble form (4) and, as described by Tanaka and colleagues (8), patients with NK lymphoma or large granular lymphocytic leukemia may release the soluble form of FasL. Once in the blood stream, these factors might trigger apoptotic death in sensitive cells. In another study it was demonstrated that tumor cells expressing FasL can induce lymphocyte apoptosis, a mechanism that possibly contributes to the tumor ability of immune evasion (9). Hahne and colleagues (10) showed that melanoma cells expressing FasL are resistant to Fas-induced apoptosis, and Keane et al. (11), using nontransformed cell lines and breast cancer cell lines, also suggested that malignant breast cells can evade apoptosis induction because of a loss of Fas system activity in cancer cells. Taken together, these proposed mechanisms may contribute to the tumor cell resistance to the immune system attack. A hypothesis currently under investigation in our laboratory is whether any of the known "death factors" is involved in the observed phenomenon. Further studies may help elucidate whether apoptosis described in peripheral blood cells from breast cancer patients is involved in the mechanisms of tumor immunological resistance.

\section{Acknowledgments}

We thank the Laboratory of Endocrinology of Hospital Pedro Ernesto for technical assistance with the cortisol determination and Dr. Jacyara M. Brito Macedo for a useful critical review of the manuscript.

\section{References}

1. Nagata S (1997). Apoptosis by death factor. Cell, 88: 355-365.

2. Abbas AK (1996). Die and let live: eliminating dangerous lymphocytes. Cell, 84: 655-657.

3. Meagher LC, Cousin JM, Seckl JR \& Haslett C (1996). Opposing effects of glucocorticoids on the rate of apoptosis in neutrophilic and eosinophilic granulocytes. J ournal of Immunology, 156: 44224428.

4. Blutler B \& Cerami A (1986). Cachetin and tumour necrosis factor as two sides of the same biological coin. Nature, 320: 584-588.

5. Segal-Bendirdjian E \& J acquemin-Sablon A (1996). Résistance au cisplatine d'une lignée de leucémie murine associée à un défaut d'appoptose. Bulletin du Cancer, 83: 371-378.

6. Wyllie AH (1992). Apoptosis and the regulation of cell numbers in normal and neoplastic tissues: an overview. Cancer and Metastasis Reviews, 11: 95-103.

7. Elston CW \& Ellis IO (1991). Pathological prognostic factors in breast cancer. I. The value of histological grade in breast cancer: experience from a large study with long-term follow-up. Histopathology, 19: 403-410.

8. Tanaka M, Suda T, Haze K, Nakamura N, Sato K, Kimura F, Motoyoshi K, Mizuki M, Tagawa S, Ohga S, Hatake K, Drummond AH \& Nagata S (1996). Faz ligand in human serum. Nature Medicine, 2: 317-322.

9. Strand S, Hofmann WJ, Hug H, Müller M,
Otto G, Strand D, Mariani SM, Stremmel W, Krammer PH \& Galle PR (1996). Lymphocyte apoptosis induced by CD95 (APO/Fas) ligand expressing tumor cells a mechanism of immune evasion. Nature Medicine, 2: 1361-1366.

10. Hahne M, Rimoldi D, Schröter M, Romero $P$, Schreier $M$, French LE, Scheineder $P$, Bornand T, Fontana A, Lienard D, Cerottini J -C \& Tschopp J (1996). Melanoma cell expression of Fas (Apo-1/CD95) ligand: implications for tumor immune escape. Science, 274: 1363-1366.

11. Keane MM, Ettenberg SA, Lowrey GA, Russell EK \& Lipkowitz S (1996). Fas expression and function in normal and malignant breast cell lines. Cancer Research, 56: 4791-4798. 